\title{
Simple coiling using single or multiple catheters without balloons or stents in middle cerebral artery bifurcation aneurysms
}

\author{
Sung-Chul Jin • O-Ki Kwon • Chang Wan Oh • \\ Jae Seung Bang • Gyojun Hwang • Nam Mi Park • \\ Eun A Jung • Moon Hee Han • Hyun-Seung Kang • \\ Hyun Park \\ Received: 17 June 2012 / Accepted: 7 November 2012 / Published online: 29 November 2012 \\ (C) The Author(s) 2012. This article is published with open access at Springerlink.com
}

\begin{abstract}
Introduction We evaluated the outcomes of middle cerebral artery bifurcation $(\mathrm{MCAB})$ aneurysms treated with simple coiling using single or multiple catheters without stents or balloons.

Methods This study included 100 patients with 103 MCAB aneurysms who underwent a simple coiling procedure without the adjuvant use of stents or balloons. The angiographic clinical outcomes and recurrence of these aneurysms were evaluated.

Results Of the 103 aneurysms, 102 (99.0\%) aneurysms were successfully treated with simple coiling. One patient died from the consequences of a procedural aneurysm rupture. The treatment-associated permanent morbidity and mortality rates were 0 and $1.0 \%$, respectively. Post-coiling angiograms showed 28 complete occlusions (27.2\%), 60 neck remnants (58.3\%), and 14 partial occlusions (13.6 \%).
\end{abstract}

\section{S.-C. Jin}

Department of Neurosurgery, Inje University,

Haeundae Paik Hospital, Busan, South Korea

O.-K. Kwon $(\varangle) \cdot$ C. W. Oh • J. S. Bang $\cdot$ G. Hwang $\cdot$

N. M. Park $\cdot$ E. A. Jung

Department of Neurosurgery, Seoul National University

Bundang Hospital, 300 Gumi-dong, Bungdang-gu,

Seongnam, Gyeonggi 463-707, South Korea

e-mail: meurokwonoki@gmail.com

\section{H. Han}

Department of Radiology, Seoul National University Hospital, Seoul, South Korea

H.-S. Kang

Department of Neurosurgery, Seoul National University Hospital, Seoul, South Korea

H. Park

Department of Neurosurgery, Gyeongsang National University Hospital, Gyeongsang National University School of Medicine, Jinju, South Korea
A follow-up angiography (median duration, 30 months; range, 3-73 months) was performed in 80 lesions. Recanalisation was found in 28 lesions (35.0\%), of which 6 were complete occlusions, 18 were neck remnants, and 4 were partial occlusions, as determined by post-coiling angiograms. Among these lesions, 14 major recurrences were retreated with coiling $(n=12)$ and clipping $(n=2)$ without complications. Age (odds ratio [OR], 0.93; $95 \%$ confidence interval $[\mathrm{CI}],-0.11$ to $-0.01 ; p=0.03)$, the presence of a rupture (OR, 3.89; $95 \% \mathrm{CI}, 0.12$ to $2.60 ; p=0.03$ ), and a wide aneurysm neck (OR, 6.40; $95 \%$ CI, 0.57 to 3.14 ; $p=0.005$ ) were significantly associated with the aneurysm recurrence, as determined by multivariable analyses.

Conclusion Our study suggests that simple coiling of $\mathrm{MCAB}$ aneurysms is feasible and safe; however, it has limitations in durability, particularly in ruptured or widenecked aneurysms and in young patients.

Keywords Aneurysm · Coiling · Simple $\cdot$ Middle cerebral artery $\cdot$ Endovascular

\section{Introduction}

Surgical clipping is a feasible and durable treatment for middle cerebral artery bifurcation (MCAB) aneurysms. In contrast, endovascular coiling is not a primary option for MCAB aneurysm treatment due to its unfavourable anatomical properties such as wide necks and the incorporation of important vessels. Advanced adjuvant devices, such as stents or balloons, have been used successfully with favourable outcomes [1-3]. Most reports have demonstrated that balloon-assisted coiling does not pose as an additive risk of thromboembolism or aneurysm perforation [4-6]. However, a few studies have indicated that balloon-assisted coiling may result in increased thromboembolisms compared to the 
single catheter technique $[7,8]$. There has been reluctance in using devices such as stents or balloons because of the potential risks associated with its use at distal cerebral vessels, which are distal to the bifurcation of the internal carotid artery (ICA). For many years, we have demonstrated that our coiling method for MCAB aneurysms is simple and conventional using single or multiple microcatheters; neither a stent nor balloons are applied.

In this report, we summarise the clinical and radiological outcomes of our study to evaluate the safety, durability, and effectiveness of simple coiling in MCAB aneurysms.

\section{Materials and methods}

\section{Patients}

We retrospectively assessed 100 patients with $103 \mathrm{MCAB}$ aneurysms who underwent a detachable coil embolisation in our institution between August 2003 and December 2008. The study was approved by the Institutional Review Board.

In our practice, ruptured $\mathrm{MCAB}$ aneurysms are treated on an emergent basis. For unruptured MCAB aneurysms, treatment was recommended in patients with aneurysms larger than $5 \mathrm{~mm}$ and if the patient was 75 years old or younger. If the MCAB aneurysms were smaller than $2 \mathrm{~mm}$, then an annual follow-up evaluation was recommended. For MCAB aneurysms with sizes between 2 and $5 \mathrm{~mm}$, the decision for treatment was dependent on the morphology of the aneurysm, such as the daughter sac, multiplicity, previous history of subarachnoid haemorrhage (SAH), patient's age, patient's emotional status, and/or technical feasibility.

The clinical data were obtained from the patient's medical records. Using procedural records and images, the aneurysm characteristics, including the location of the aneurysm, size of the aneurysm (width and height), aneurysm neck diameter, vessel incorporation, and procedural details, including the immediate angiographic results and complications, were reviewed.

\section{Endovascular treatment}

Coiling is the primary treatment option considered when a patient refuses surgery or displays poor medical conditions. Coiling is not attempted when the aneurysm height is less than $2 \mathrm{~mm}$ or when the major branch is incorporated into the aneurysm dome. During the coiling procedure, none of the patients were treated with stent- or balloon-assisted coiling. A simple coiling method using single or multiple microcatheters was performed. A multiple microcatheter technique was applied for aneurysms with broad necks or incorporated vessels, and this operational procedures have been previously described $[9,10]$. All of the procedures were performed under general anaesthesia using a biplane system (Integris Allura,
Phillips Medical Systems, Netherlands). For unruptured aneurysms, the patients received an intravenous bolus injection of 3,000-U heparin, and 1,000-U heparin was given every hour thereafter. Heparin was not given in cases of acute subarachnoid haemorrhage (SAH).

Angiographic findings, including the determination of aneurysm size, degree of occlusion, and recurrence, were interpreted by a neurointerventionist (S-C. J.) who was blinded to the clinical and procedural data. The aneurysm size was measured using three-dimensional angiographic images. The aneurysm width was determined by measuring the longest diameter of the fundus parallel to the axis of the aneurysm neck. The height was determined as the longest diameter of the fundus vertical to the axis of the aneurysm neck. The angiographic outcomes were classified as a complete occlusion, residual neck, or partial occlusion according to the Raymond scale [11]. The aneurysm recurrence was defined as a new opacification of the aneurysm sac or as an increase in the size of the residual neck or sac, which was further classified into two subcategories: minor recurrence, which did not require retreatment, or major recurrence, which required retreatment.

Follow-up imaging was performed. The time-of-flight and contrast-enhanced MR angiographies (MRA) were performed. A digital subtraction angiography (DSA) was performed when major recanalisation was suspected on the basis of the follow-up MRA studies. Retreatment was considered when the major recanalisation was confirmed on the DSA. A trained physician's assistant performed a clinical assessment on the patients at the time of discharge and at the outpatient clinic after discharge. The Modified Glasgow Outcome Scale (GOS) scores, which were categorised into good (GOS 4 to 5) and poor (GOS 1 to 3) functional outcomes, were used to evaluate the clinical outcomes [12].

\section{Statistical analysis}

A statistical analysis was performed to evaluate the factors that could affect the aneurysm recurrence with the aid of the medical research collaborating centre. Nominal data were analysed using $\chi^{2}$ or Fisher's exact test and the numerical data with Student's $t$ test or Mann-Whitney's $U$ test, when appropriate. A two-tailed $p<0.05$ was defined as statistically significant. Multivariable analysis using a generalised estimation equation was performed to determine the independent factors of the aneurysm recurrence. Variables were selected for entry into the multivariable model on the basis of the results of the backward selection method. Using this approach, the model was fitted with all of the variables of interest (univariate analyses, $p<0.1$ ). Next, the least significant variable was removed if it was not significant at the selected critical level. We continued by successively refitting the reduced models and applying the same rule until all of the remaining variables were statistically significant. 
The odds ratios and $95 \%$ confidence intervals were then calculated.

\section{Results}

\section{General characteristics}

Of the analysed 100 patients with 103 MCAB aneurysms, 39 $(39.0 \%)$ patients were male. The median age was 60.5 years (mean $\pm \mathrm{SD}, 58.6 \pm 13.1$ years; range, $17-83$ years). Fiftyeight $(58.0 \%)$ patients presented with SAH. The Hunt and Hess grades of the patients were as follows: grade II in 35 patients $(60.3 \%)$, grade III in 9 patients $(15.5 \%)$, grade IV in 10 patients $(17.2 \%)$, and grade $\mathrm{V}$ in 4 patients $(6.9 \%)$. The Fisher grades of the patients included grade I in 3 patients (5.2\%), grade II in 6 patients $(10.3 \%)$, grade III in 23 patients $(39.7 \%)$, and grade IV in 26 patients $(44.8 \%)$. The mean height, width, and neck size of the aneurysms were $5.1 \pm 2.8$, $5.2 \pm 2.7$, and $3.5 \pm 1.6 \mathrm{~mm}$, respectively. A broad aneurysm neck larger than $4 \mathrm{~mm}$ was observed in 31 patients (30.1\%), and 43 aneurysms (41.7\%) had incorporated branch arteries (M2) around the aneurysm necks.

\section{Procedural outcome and complications}

The single conventional catheter and multiple microcatheter techniques were used in 58 (56.3 \%) and 45 (43.7 \%) aneurysms, respectively. A successful coil embolisation was achieved in $102(99.0 \%)$ lesions: (1) a complete occlusion in 28 aneurysms $(27.2 \%)$, (2) neck remnant in 60 aneurysms (58.3\%), and (3) partial occlusion in 14 aneurysms (13.6\%).

Fifteen (14.6\%) procedure-related adverse events occurred, including a procedural aneurysmal rupture in four aneurysms (3.9\%) and thromboembolism in ten aneurysm (9.7\%) cases. For ten cases with thromboembolic complications, intraarterial thrombolysis using urokinase, abciximab, or tirofiban was performed in seven cases. A partial or complete recanalisation was achieved in all of the lesions. Most of the complications did not result in a neurological deficit. A procedurerelated mortality developed in one $(1.0 \%)$ lesion with a procedural rupture. This patient with Hunt and Hess grade IV SAH developed a contrast leakage during the coiling procedure. Further packing of the aneurysm resulted in a complete occlusion; however, the patient died of intractable increased intracranial pressure despite a decompressive craniectomy.

\section{Clinical outcomes}

Of the 58 patients with acute SAHs, 42 patients $(72.4 \%)$ showed favourable outcomes (GOS score $\geq 4$ ) at discharge. Nine $(15.5 \%)$ of the 58 patients with SAH continued to show a moderate or severe disability (GOS score $>1$ and $\leq 3$ ) mainly due to an initially poor clinical status. Seven $(12.1 \%)$ patients died for the following reasons: (1) intractable intracranial pressure caused by an initial haemorrhage including a large cerebral haematoma $(n=5)$, (2) major infarction due to a severe vasospasm $(n=1)$, and (3) aneurysm rupture during the procedure $(n=1)$. Of the 42 patients with unruptured MCA bifurcation aneurysms, all of the patients remained clinically intact.

Eighty-four patients were evaluated for a median duration of 29.5 months (range, 3 to 78 months). In the SAH group, 40 patients $(68.9 \%)$ exhibited favourable clinical outcomes. Four patients' severe or moderate disability (GOS score 2 or 3 ) remained $(n=3)$ or was aggravated $(n=1)$. All of the 40 patients with unruptured MCA bifurcation aneurysms maintained good clinical outcomes.

An aneurysm rupture occurred in two patients after the initial procedure. Coiling was performed on one patient with an unruptured MCA aneurysm using the multiple catheter technique and resulted in a neck remnant on the final angiogram. The patient developed SAH 17 months after the initial coiling and then underwent re-coiling, which resulted in no further disabilities. Coiling was performed on the other patient with a ruptured MCA aneurysm using a single catheter technique, which resulted in a neck remnant on the final angiogram. The patient had suffered from SAH for 6 months after the initial coiling and then underwent re-coiling with moderate disability (GOS 3).

\section{Follow-up angiographic outcomes}

A follow-up angiography was performed in 77 patients with 80 aneurysms (77.7 \%). The follow-up loss was mainly due to the patients' grave conditions. The median time from the coiling procedure to the follow-up angiography was 30.0 months (range, 3 to 73 months).

A recurrence of the aneurysm neck or sac was found in $28(35.0 \%)$ lesions. Retreatment was performed in 14 lesions $(17.5 \%)$, and the median time until retreatment was 14 months (range, 2-63 months), (1) within 6 months after coiling $(n=3)$, (2) between 6 months and 1 year after coiling ( $n=2)$, (3) between 1 and 2 years after coiling $(n=7)$ and (4) beyond 2 years after the procedure $(n=2)$. Two of the patients were retreated by surgical clipping.

The aneurysm recurrence was significantly different according to the size of the aneurysm neck $(p=0.019)$, patient's age $(p=0.036)$, and rupture $(p=0.001)$. However, the recurrence rate did not differ according to the coiling techniques, packing density of the coils, presence of vessel incorporation, initial angiographic result, or width or height of the aneurysm (Table 1). In a multivariable analysis, young age (OR, $0.93 ; 95 \% \mathrm{CI},-0.11$ to $-0.01 ; p=0.03$ ) and aneurysms with a ruptured (OR, 3.89; CI, 0.12 to 2.60; $p=0.03$ ) or wide neck (OR, 6.40; $95 \% \mathrm{CI}, 0.57$ to 3.14; 
Table 1 Univariate analysis of recurrence in the 80 MCA bifurcation aneurysms followed

\begin{tabular}{|c|c|c|c|}
\hline Characteristics & No recurrence & Recurrence & $p$ value \\
\hline Aneurysm height (mm) & & & 0.099 \\
\hline$<7$ & 47 & 21 & \\
\hline$\geq 7$ & 5 & 7 & \\
\hline Aneurysm width (mm) & & & 0.766 \\
\hline$<7$ & 43 & 22 & \\
\hline$\geq 7$ & 9 & 6 & \\
\hline Aneurysm neck (mm) & & & 0.019 \\
\hline$<4$ & 42 & 15 & \\
\hline$\geq 4$ & 10 & 13 & \\
\hline Relationship with vessel & & & 0.197 \\
\hline No incorporation & 32 & 13 & \\
\hline Incorporation $^{\mathrm{a}}$ & 20 & 15 & \\
\hline Presence of rupture & & & 0.001 \\
\hline Yes & 20 & 22 & \\
\hline No & 32 & 6 & \\
\hline Age (years) & & & 0.036 \\
\hline$<60$ & 24 & 20 & \\
\hline$\geq 60$ & 28 & 8 & \\
\hline Embolisation technique & & & 0.512 \\
\hline Single catheter technique & 30 & 14 & \\
\hline Multiple catheter technique & 22 & 14 & \\
\hline Angiographic results & & & 0.439 \\
\hline Complete & 16 & 6 & \\
\hline Incomplete & 36 & 22 & \\
\hline Packing density of coils & $29.9 \pm 14.3$ & $26.7 \pm 9.7$ & 0.428 \\
\hline
\end{tabular}

${ }^{a}$ Incorporation means that vessel branches originate from the aneurysm

$p=0.005)$ were independently associated with recurrence (Table 2).

\section{Discussion}

In this study, most of the MCA bifurcation aneurysms were coiled without serious complication using the single or multiple catheter technique. This low procedural morbidity and mortality may be related to the indication we use for coiling. For broad-necked or vessel-incorporated aneurysms, a multiple catheter technique was useful. A procedural complication that resulted in mortality occurred in one patient with a ruptured aneurysm (1\%). There was no procedural morbidity or mortality for the unruptured lesions. In the current literature, the morbidity and mortality rates associated with surgical clipping were 4.2-12.9 and 6.5-10\%, respectively, for ruptured MCA aneurysms [13-15]. For unruptured lesions, the reported morbidity rates were approximately $3 \%[16,17]$. Our study suggests that simple coiling using single or multiple catheters without the use of stents or balloons may be performed with acceptable risks for MCA bifurcation aneurysms with favourable configurations. However, our study also shows that simple coiling using single or multiple catheters for MCAB aneurysms has cardinal limitations in the degree of occlusion and durability.

Our results showed that a partial occlusion was $14.3 \%$, which may be higher compared to previous studies [1-3]. For ruptured aneurysms, a partial occlusion may have a risk of re-bleeding.

The recurrence rate in this study was $35 \%$. Half of the patients required retreatment, which included surgical clipping. In our study, the overall retreatment rate of the coiled aneurysms except that of MCAB aneurysms was approximately $7 \%$ (unpublished data). For the MCAB aneurysms, the mean follow-up duration was 30.0 months; however, the recurrence rates could be higher with a longer follow-up evaluation period.

Thromboembolism (9.7\%) developed mostly in the ruptured aneurysms, and the rate of thromboembolism was higher compared to other studies [1-3]. The high thromboembolism observed in this study may be related to the absence of heparinisation in the coiling of the ruptured aneurysms. Recently, however, significant thromboembolism, which was required for IA thrombolysis, developed in less than $1 \%$ of the patients (unpublished data) during the coiling of ruptured aneurysms. Thus, we suggested that early experience with coiling may contribute to the relatively high rate of thromboembolism.

In this study, we did not use stent- or balloon-assisted techniques. Several reports have previously demonstrated that
Table 2 Multivariable analysis using GEE for evaluating variables related with recurrence in the 80 MCA bifurcation aneurysms followed

$O R$ odds ratio, $C I$ confidential interval

\begin{tabular}{lcccccc}
\hline Parameter & Estimate & Standard Error & OR & $95 \%$ CI & & $p$ value \\
\hline Intercept & 4.4336 & 1.7235 & & 1.0555 & 7.8116 & 0.010 \\
Age & -0.0579 & 0.0268 & 0.9333 & -0.1104 & -0.0054 & 0.031 \\
Presence of rupture & & & & & \\
Non-rupture & -1.3591 & 0.631 & 1 & -2.5959 & -0.1223 & 0.031 \\
Rupture & 0 & 0 & 3.8927 & 0 & 0 &. \\
Aneurysm neck $(\mathrm{mm})$ & -1.8566 & 0.6563 & 1 & -3.143 & -0.5702 & 0.005 \\
$<4$ & 0 & 0 & 6.4019 & 0 & 0 &. \\
$\geq 4$ & & & & & & \\
\end{tabular}


stents or balloons may be successfully applied to treat MCA bifurcation aneurysms [3, 18-21]; however, we were very reluctant to use these devices because of concerns regarding the potential risks of unexpected adverse events such as thromboembolism or aneurysm perforation distal to the bifurcation of ICA. Surgical risks for MCAB aneurysms were not very high. Until now, there have been no data on the outcomes of stentassisted coiling in MCAB aneurysms. Several reports have demonstrated that the procedural complication rates of the stent-assisted technique for MCAB aneurysms are not higher compared to simple coiling and showed favourable durability $[22,23]$. As our study suggests, simple coiling using single or multiple catheters has a high recurrence rate in MCAB aneurysms. The stent-assisted technique may be an alternative option to enhance durability in the coiling of MCAB aneurysms.

Our multivariable analysis results demonstrate that age, rupture, and wide neck characteristics are significant predicting factors of aneurysm recurrence. Several other studies have also shown rupture and wide neck as predicting factors for recurrence [1, 24-26]. In our study, we also identified age as an influencing factor for MCA bifurcation aneurysm recurrence. Moreover, recurrence occurred more often in young patients. This result may be related to the decreased elasticity and compliance in the vascular wall with increasing age, both of which contribute in the prevention of recanalisation in the treatment of the aneurysm sac.

\section{Conclusions}

Simple coiling without a stent or a balloon is a feasible treatment method for MCAB aneurysms. However, the recurrence rate was high. More than one third of the aneurysms recurred. Approximately half of the recurred aneurysms required retreatment. Our study suggests that simple coiling of MCA bifurcation aneurysms is feasible but that it has a limited durability.

Acknowledgments This study was supported by the SNUBH Research Fund (03-2012-010).

Conflict of interest We declare that we have no conflict of interest.

Open Access This article is distributed under the terms of the Creative Commons Attribution License which permits any use, distribution, and reproduction in any medium, provided the original author(s) and the source are credited.

\section{References}

1. Vendrell JF, Menjot N, Costalat V et al (2009) Endovascular treatment of 174 middle cerebral artery aneurysms: clinical outcome and radiologic results at long-term follow-up. Radiology 253:191-198
2. Quadros RS, Gallas S, Noudel R, Rousseaux P, Pierot L (2007) Endovascular treatment of middle cerebral artery aneurysms as first option: a single center experience of 92 aneurysms. Am J Neuroradiol 28:1567-1572

3. Iijima A, Piotin M, Mounayer C, Spelle L, Weill A, Moret J (2005) Endovascular treatment with coils of 149 middle cerebral artery berry aneurysms. Radiology 237:611-619

4. Shapiro M, Babb J, Becske T, Nelson PK (2008) Safety and efficacy of adjunctive balloon remodeling during endovascular treatment of intracranial aneurysms: a literature review. Am J Neuroradiol 29:1777-1781

5. Layton KF, Cloft HJ, Gray LA, Lewis DA, Kallmes DF (2007) Balloon-assisted coiling of intracranial aneurysms: evaluation of local thrombus formation and symptomatic thromboembolic complications. Am J Neuroradiol 28:1172-1175

6. Cekirge HS, Yavuz K, Geyik S, Saatci I (2011) HyperForm balloon remodeling in the endovascular treatment of anterior cerebral, middle cerebral, and anterior communicating artery aneurysms: clinical and angiographic follow-up results in 800 consecutive patients. J Neurosurg 114:944-953

7. Sluzewski M, Bosch JA, van Rooij WJ, Nijssen PC, Wijnalda D (2001) Rupture of intracranial aneurysms during treatment with Guglielmi detachable coils: incidence, outcome, and risk factors. J Neurosurg 94:238-240

8. Sluzewski M, van Rooij WJ, Beute GN, Nijssen PC (2006) Balloonassisted coil embolization of intracranial aneurysms: incidence, complications, and angiography results. J Neurosurg 105:396-399

9. Kwon OK, Kim SH, Oh CW et al (2006) Embolization of widenecked aneurysms with using three or more microcatheters. Acta Neurochir (Wien) 148:1139-1145

10. Kwon OK, Kim SH, Kwon BJ et al (2005) Endovascular treatment of wide-necked aneurysms by using two microcatheters: techniques and outcomes in 25 patients. Am J Neuroradiol 26:894-900

11. Roy D, Milot G, Raymond J (2001) Endovascular treatment of unruptured aneurysms. Stroke 32:1998-2004

12. Teasdale G, Jennett B (1974) Assessment of coma and impaired consciousness. A practical scale. Lancet 2:81-84

13. Heros RC, Fritsch MJ (2001) Surgical management of middle cerebral artery aneurysms. Neurosurgery 48:780-785

14. Rinne J, Hernesniemi J, Niskanen M, Vapalahti M (1996) Analysis of 561 patients with 690 middle cerebral artery aneurysms: anatomic and clinical features as correlated to management outcome. Neurosurgery 38:2-11

15. Suzuki J, Yoshimoto T, Kayama T (1984) Surgical treatment of middle cerebral artery aneurysms. J Neurosurg 61:17-23

16. Yasui N, Nishimura H (2004) Surgical treatment of unruptured intracranial aneurysms over the past 22 years. Neurol Med Chir (Tokyo) 44:155-161

17. Regli L, Uske A, de Tribolet N (1999) Endovascular coil placement compared with surgical clipping for the treatment of unruptured middle cerebral artery aneurysms: a consecutive series. J Neurosurg 90:1025-1030

18. Pumar JM, Lete I, Pardo MI, Vazquez-Herrero F, Blanco M (2008) LEO stent monotherapy for the endovascular reconstruction of fusiform aneurysms of the middle cerebral artery. Am J Neuroradiol 29:1775-1776

19. Pero G, Denegri F, Valvassori L, Boccardi E, Scialfa G (2006) Treatment of a middle cerebral artery giant aneurysm using a covered stent. Case report. J Neurosurg 104:965-968

20. Doerfler A, Wanke I, Goericke SL et al (2006) Endovascular treatment of middle cerebral artery aneurysms with electrolytically detachable coils. Am J Neuroradiol 27:513-520

21. Sani S, Lopes DK (2005) Treatment of a middle cerebral artery bifurcation aneurysm using a double neuroform stent "Y" configuration and coil embolization: technical case report. Neurosurgery 57:E209 
22. Yang P, Liu J, Huang Q et al (2010) Endovascular treatment of wide-neck middle cerebral artery aneurysms with stents: a review of 16 cases. Am J Neuroradiol 31:940-946

23. Pierot L, Cognard C, Anxionnat R, Ricolfi F (2011) Remodeling technique for endovascular treatment of ruptured intracranial aneurysms had a higher rate of adequate postoperative occlusion than did conventional coil embolization with comparable safety. Radiology 258:546-553

24. Raymond J, Guilbert F, Weill A et al (2003) Long-term angiographic recurrences after selective endovascular treatment of aneurysms with detachable coils. Stroke 34:1398-1403

25. Campi A, Ramzi N, Molyneux AJ et al (2007) Retreatment of ruptured cerebral aneurysms in patients randomized by coiling or clipping in the International Subarachnoid Aneurysm Trial (ISAT). Stroke 38:1538-1544

26. Fernandez Zubillaga A, Guglielmi G, Vinuela F, Duckwiler GR (1994) Endovascular occlusion of intracranial aneurysms with electrically detachable coils: correlation of aneurysm neck size and treatment results. Am J Neuroradiol 15:815-820 